\title{
Burnout y factores estresantes en profesionales sanitarios de las unidades de cuidados intensivos
}

\author{
Rafael Ballester-Arnal ${ }^{1}$, Sandra Gómez-Martínez ${ }^{1,2}$, Beatriz Gil-Juliá1, \\ M. Desamparados Ferrándiz-Sellés ${ }^{1,3}$ y Eladio J. Collado-Boira ${ }^{1}$ \\ ${ }^{1}$ Universidad Jaime I, Castellón, España \\ ${ }^{2}$ Universidad Internacional de Valencia, Valencia, España \\ ${ }^{3}$ Hospital General Universitario de Castellón, Castellón, España
}

\begin{abstract}
Resumen: El objetivo del presente estudio fue estudiar el burnout y los factores que provocan estrés en los profesionales que trabajan en las unidades de cuidados intensivos (UCI). Participaron en el estudio 117 sanitarios que respondieron a un registro sociodemográfico, al cuestionario Maslach Burnout Inventory (MBI) y al Cuestionario de Factores Estresantes en UCI. Los factores más estresantes eran la sobrecarga de trabajo y la falta de personal. Más del $30 \%$ de la muestra presentaba puntuaciones elevadas en alguna subescala de burnout. Encontramos diferencias en función del género (los hombres presentaban mayor Despersonalización). Los análisis de regresión mostraron que el género y la experiencia profesional podrían predecir el burnout. Este trabajo arroja información valiosa acerca de la necesidad de realizar programas específicos que proporcionen a los sanitarios de las UCIs estrategias que permitan la prevención de aspectos que pueden influir en su estado emocional.
\end{abstract}

Palabras clave: Burnout; profesionales sanitarios; estrés laboral; estrés psicológico; unidades de cuidados intensivos.

\section{Burnout and stressors in intensive care units' health workers}

\begin{abstract}
The need arises to study burnout and stress factors in intensive care units (ICU) health workers, because of the characteristics of their work environment. To achieve this aim, 117 health workers responded to the Demographic Questionnaire, the Maslach Burnout Inventory (MBI), and the ICU Stress Factors Questionnaire. The issues causing higher levels of stress were overload and understaffing. More than $30 \%$ of the sample presented elevated scores on any burnout subscale. Gender differences were found, with men presenting greater depersonalization. Regression analysis showed that gender and professional experience could predict burnout. This paper provides valuable information about the need for specific programs providing ICU healthcare professionals with strategies that allow prevention of issues that can influence their emotional state.
\end{abstract}

Keywords: Burnout; health workers; work stress; psychological stress; intensive care units.

\section{Introducción}

Recibido: 08 marzo 2016; aceptado 12 de julio 2016

Correspondencia: Rafael Ballester-Arnal, Facultad de Psicología, Universidad Jaime I, Avda. Vicent Sos Baynat s/n, 12071, Castellón de la Plana, España. Correo-e: rballest@uji.es

Agradecimientos: Este proyecto se ha realizado con la colaboración del Servicio de Medicina Intensiva de un hospital de la Comunidad Valenciana y ha recibido financiación de la Conselleria de Sanitat de la Generalitat Valenciana (Ref. AP-040/08) y del Ministerio de Educación y Ciencia (PSI 2008- 01642/PSIC). Expresamos nuestro agradecimiento al Dr. Abizanda con el que iniciamos esta línea de trabajo y a todos los profesionales sanitarios que han participado en el estudio.
Las unidades de cuidados intensivos (UCI) son, por sus características, lugares de trabajo estresantes. Los profesionales que trabajan en estas Unidades se enfrentan a diario a situaciones en las que predominan la enfermedad grave, el dolor, la muerte y la necesidad de tomar decisiones inmediatas en cuestiones de vital importancia. Todo ello, acompañado de otros aspectos como el sentimiento de frustración por no poder conseguir siempre el restablecimiento de la salud de los enfermos y la implicación personal con los pacientes. Algunos estu- 
dios como el de Ballester, Gómez, Gil y Abizanda (2015) han mostrado la presencia de depresión y ansiedad en los profesionales sanitarios, existiendo diferencias en función del género y del puesto laboral. A esto hay que añadirle otros estresores del ambiente y la organización del trabajo como la escasa formación en el reconocimiento y control de las emociones, el conflicto y la ambigüedad de rol, el horario de trabajo, la falta de cohesión en los equipos y la burocratización de las instituciones sanitarias (Ortega y López, 2004). Por ello, surge la necesidad de estudiar el burnout y los factores que más estrés provocan en estos profesionales con el fin de intentar mejorar su calidad de vida. El burnout se define como un síndrome compuesto por tres dimensiones: el agotamiento emocional (desgaste, pérdida de energía o cansancio que puede manifestarse de manera física y/o psicológica); la despersonalización (cambio negativo en la relación con otras personas, desarrollándose sentimientos, actitudes y respuestas negativas, distantes y frías, especialmente hacia los usuarios que pueden ser considerados como meras enfermedades o casos clíni$\cos$ ); y la baja realización personal (tendencia a evaluarse a uno mismo y al propio trabajo de forma negativa como consecuencia de la cual puede aparecer irritabilidad, evitación de las relaciones profesionales, baja productividad, incapacidad para soportar la tensión, pérdida de la motivación hacia el trabajo, sentimientos de fracaso y baja autoestima) (Maslach y Jackson, 1986).

Este síndrome puede tener consecuencias psicológicas negativas pero además, se ha constatado que tiene un impacto negativo en la salud física. De hecho, en los últimos años, se ha empezado a estudiar la utilidad de los marcadores biológicos relacionados con el burnout pues facilitarían la prevención y la detección precoz de este síndrome (Gómez-Alcaina, Montero-Marín, Demarzo, Pereira y García-Campayo, 2013). El burnout ha sido asociado a un elevado riesgo de enfermedades cardiovasculares (Grossi, Perski, Evengard, Blomkvist y Orth-Gomér, 2003), a una peor respuesta inmunológica (Bargellini, et al., 2000) y a la presencia de trastornos psicosomáticos, por ejemplo, de tipo gastrointestinal (Fernández, 2000). A ello se añaden los comportamientos mediante los que se intenta reducir la ansiedad y que, a la vez, median en el deterioro de la calidad de vida, como las conductas adictivas.

En los últimos años, la investigación sobre el burnout se ha ido popularizando en todos los países del mundo y se han realizado estudios relacionados con profesionales de la salud (Santos y Abalo, 2014) como en otros colectivos (Bresó, Caballero y González, 2015; Esteras, Chorot, y Sandín, 2014; Jiménez, Lara, Muñoz, Chavez, y Loo, 2014). Por su parte, los equipos de profesionales de las UCI han sido especialmente analizados en los estudios internacionales sobre burnout y factores estresantes. Así, el trabajo llevado a cabo por Poncet et al. (2007), en el que se evaluó a más de 2000 enfermeros de UCI, mostró que un tercio de éstos presentaba síntomas graves de burnout. Se concluía que los conflictos entre enfermeros y médicos contribuían a aumentar los síntomas, mientras que el hecho de participar en grupos de investigación les protegía. En otro estudio, desarrollado por Embriaco et al. (2007), casi la mitad de los 978 médicos intensivistas informaron altos niveles de burnout, estando los factores organizacionales (tipo de UCI, número de camas, pacientes ingresados, horario, absentismo, relación con los compañeros, etc. muy relacionados con estos niveles. En un estudio desarrollado únicamente con enfermeras por Verdon, Merlani, Perneger y Ricou (2008) su prevalencia fue menor $(28 \%$ de 107 participantes). Esto iba en la línea de los resultados de Raggio y Malacarne (2007), quienes concluyeron que los médicos de UCI padecían más síntomas que el personal de enfermería, siendo éstos distintos entre hombres y mujeres. Así, los síntomas de cansancio emocional fueron más frecuentes en médicos mujeres, mientras que los síntomas de despersonalización fueron más frecuentes en médicos hombres y los síntomas de la falta de realización personal fueron más frecuentes en los enfermeros hombres. Embriaco et al (2012) mostraron que las personas con niveles más altos de burnout, presentaban síntomas de depresión, mientras que Quenot et al. (2012), concluyen que, tras implementar una estrategia de comunicación con respecto a las prácticas médicas al final de la vida en la que se le daba prioridad a la disminución del sufrimiento tanto de las familias como de los profesionales, se redujo del riesgo de burnout del 50\%.

Existe escasa evidencia sobre si el síndrome de burnout, además de tener consecuencias personales y profesionales para los sanitarios, puede afectar a la seguridad del paciente y de qué manera. No obstante, la literatura apunta a que algunas consecuencias del burnout como los síntomas de fatiga física y emocional, la depresión o las dificultades de concentración pueden tener un papel negativo en el rendimiento en entornos donde, como en UCI, son necesarios altos niveles de atención y motivación durante un periodo largo de tiempo (Reader, $\mathrm{Cu}$ therson y Decruyanier, 2008). Además, una cuestión que complica el panorama es que el personal médico tiende a negar las vulnerabilidades personales. A este respecto, Sexton, Thomas y Helmreich, (2000) concluyeron que, en comparación con los pilotos de aviación, los médicos de cuidados intensivos son mucho más propensos a informar de que están capacitados para desempeñar eficazmente su trabajo en momentos críticos, aunque estén 
cansados. Por ello es importante conocer y abordar los síntomas de burnout y los factores estresantes de estos profesionales puesto que, no solamente afectan al propio profesional, sino que pueden tener consecuencias a nivel asistencial, tanto en la calidad del cuidado a los pacientes como en la relación con los familiares de éstos.

Dada la escasez de investigaciones realizadas en España sobre el burnout en médicos y personal de enfermería (enfermeras/os y auxiliares), nos planteamos el objetivo de estudiar la asociación entre los factores estresantes y los síntomas de burnout en estos profesionales, así como también examinar su posible relación con las variables sociodemográficas y laborales.

\section{Método}

\section{Participantes}

Los participantes fueron 117 profesionales de la UCI del Hospital General Universitario de Castellón. La edad media era de 38.02 años $(D T=8.89)$ y el $74.1 \%$ eran mujeres. Por lo que respecta a las características laborales, el $28.4 \%$ eran médicos, el $49.1 \%$ enfermeros y el $22.4 \%$ auxiliares, y la media de experiencia era de 12.4 años $(D T=8.2)$ en la profesión y de 7.1 años $(D T=$ 6.84) en la UCI.

\section{Instrumentos}

Hoja de registro: En esta hoja se recogían diversos datos tanto personales como relacionados con el trabajo.

Cuestionario de Factores Estresantes-UCI: Este instrumento (Ballester, Edo e Ibáñez) es una adaptación al contexto de UCI de la Escala de Estresores Hospitalarios de Richart, Cabrero y Reig (1993) para evaluar el grado de malestar asociado a diferentes estresores durante la hospitalización en pacientes médicos y quirúrgicos. El cuestionario está formado por 40 ítems referentes al estrés asociado a diversos aspectos relacionados con la UCI. Estos ítems se responden en una escala tipo Likert con cinco opciones de respuesta sobre el grado de estrés (nada, algo, bastante, mucho y muchísimo). En la versión de sanitarios se recogen aspectos sobre la toma de decisiones, la relación con pacientes y familiares, los factores organizacionales, las tareas diarias o el manejo de situaciones complejas. Finalmente se pregunta por el grado de estrés general en una escala de 0 a 4 . La fiabilidad (alfa de Cronbach) de la escala de profesionales del estudio original fue de .90 (Richart et al., 1993); en nuestro estudio fue de .95 .

Maslach Burnout Inventory (MBI; Maslach y Jackson, 1986). El MBI fue desarrollado por Maslach y
Jackson y validada en español por Gil-Monte (1994). El cuestionario consta de 16 ítems que se distribuyen en tres subescalas: Agotamiento emocional, Despersonalización y Realización personal. Los ítems se valoran en una escala tipo Likert entre 0 (nunca) y 6 ( todos los días) en función de la frecuencia con la que el encuestado ha experimentado la sensación. Se determinaron unos rangos de puntuaciones totales para cada una de las subescalas (Tabla 1). Una persona con un alto nivel de burnout sería aquella con bajas puntuaciones en Realización personal y altas en Despersonalización y Agotamiento emocional. Los valores de fiabilidad de las escalas (alfa de Cronbach) para el cuestionario original fueron de .89 para la subescala de Agotamiento emocional, .77 para la Despersonalización y .74 para la Realización personal (Maslach y Jackson, 1986). Por su parte, la adaptación española obtuvo unos índices de fiabilidad de .87 para Agotamiento emocional, .57 para Despersonalización y .72 para Realización personal en el trabajo, (Gil-Monte y Peiró, 1999). Finalmente los resultados del alfa de Cronbach en el presente estudio fueron .85 para Realización personal, .83 para Agotamiento emocional y .74 para Despersonalización.

Tabla 1. Baremo de puntuaciones del cuestionario MBI según subescalas

\begin{tabular}{lccc}
\hline & Bajo & Medio & Alto \\
\hline Agotamiento emocional & $<18$ & $19-26$ & $>27$ \\
Despersonalización & $<5$ & $6-9$ & $>10$ \\
Realización personal & $>40$ & $34-39$ & $<33$ \\
\hline
\end{tabular}

\section{Procedimiento}

Se entregó individualmente un sobre con la batería de cuestionarios a cada uno de los profesionales que trabajaban en la UCI y, para respetar la confidencialidad, se les pidió que una vez contestados todos los cuestionarios cerraran el sobre y lo depositaran en un lugar habilitado para ello situado en la secretaría de la unidad. En el sobre, se incluía una Hoja de Consentimiento Informado. Por otro lado, el proyecto de investigación del que se deriva este estudio fue aprobado por el Comité Bioético del Hospital y también por la Comisión Deontológica de la Universidad.

\section{Análisis de datos}

Los análisis se llevaron a cabo con el SPSS 19. Se realizaron análisis descriptivos y de frecuencias para la 
Tabla 2. Medias y desviaciones típicas de las puntuaciones del Cuestionario de Factores Estresantes (excepto el ítem de estrés global)

\begin{tabular}{|c|c|c|}
\hline Ítem & $M$ & $D T$ \\
\hline 4. Sobrecarga o saturación de trabajo & 2.88 & 1.1 \\
\hline 18. Falta o insuficiencia de recursos humanos para cubrir las necesidades de la unidad & 2.37 & 1.2 \\
\hline 2. Consecuencias que se puedan derivar de la rapidez en la toma de decisiones & 2.31 & 0.97 \\
\hline 5. Presión temporal en el trabajo a realizar diariamente & 2.27 & 1.23 \\
\hline 22. Falta de descansos reglados durante la jornada laboral & 2.17 & 1.19 \\
\hline 1. Necesidad de dar respuesta inmediata a situaciones complejas & 2.10 & 0.98 \\
\hline 21. Desvalorización de la profesión & 2.05 & 1.26 \\
\hline 9. Horarios de trabajo (turnos de noche o guardias) & 1.97 & 1.29 \\
\hline 24. Elevadas exigencias & 1.91 & 1.19 \\
\hline 20. Necesidad de responder a situaciones críticas con incertidumbre & 1.90 & 1.07 \\
\hline 29. Situaciones de incertidumbre porque la información de la que se dispone no es suficiente & 1.89 & 1.01 \\
\hline 16. Riesgo de incurrir en una mala praxis & 1.88 & 1.22 \\
\hline 28. Consecuencias derivadas de las decisiones que se toman & 1.88 & 0.99 \\
\hline 3. Exceso de responsabilidad para el puesto que desempeña & 1.85 & 1.12 \\
\hline 13. Gran estrés y sobrecarga emocional & 1.81 & 1.22 \\
\hline 38. Estilos de dirección y supervisión inadecuados & 1.66 & 1.16 \\
\hline 30. Sufrir agresiones de tipo verbal & 1.64 & 1.24 \\
\hline 25. Creciente complejidad de las tareas médicas y enfermeras & 1.62 & 1.05 \\
\hline 32. Falta de coordinación con otros servicios & 1.61 & 0.85 \\
\hline 8. Gravedad de los enfermos con los que se trabaja & 1.59 & 1.22 \\
\hline 14. Sensación de falta de control sobre el contenido y la planificación del trabajo & 1.59 & 1.15 \\
\hline 19. Falta o insuficiencia de recursos materiales para cubrir las necesidades del servicio & 1.58 & 1.15 \\
\hline 34. Falta de seguridad en el empleo & 1.51 & 1.16 \\
\hline 36. Interferencias con otro tipo de tareas & 1.50 & 0.97 \\
\hline 17. Contacto diario con el dolor, el sufrimiento y la muerte & 1.49 & 1.01 \\
\hline 10. Necesidad de formación continuada (manejo de aparatos, medicaciones) & 1.47 & 1.15 \\
\hline 33. Retraso en la recepción de resultados & 1.44 & 0.9 \\
\hline 26. Problemas jerárquicos entre categorías profesionales & 1.40 & 1.17 \\
\hline 35. Fluidez de las comunicaciones & 1.40 & 0.99 \\
\hline 23. Aplicación y conocimiento de nuevas tecnologías & 1.37 & 0.96 \\
\hline 27. Información sobre el estado del paciente fluctuante & 1.36 & 1.01 \\
\hline 12. Falta de delimitación en el desempeño de tareas relacionado con el puesto de trabajo que ocupa & 1.34 & 1.1 \\
\hline 15. Exposición a riesgos y accidentes laborales & 1.27 & 1.11 \\
\hline 37. Necesidad de consultar antes de tomar una decisión & 1.20 & 1.05 \\
\hline 31. Complejidad de la información a trata & 1.18 & 0.89 \\
\hline 11. Rellenar a diario múltiples hojas de registros, solicitud de pruebas, redactar informes de ingreso y alta. & 1.09 & 0.98 \\
\hline 39. Falta de adaptación al puesto & 0.89 & 0.99 \\
\hline 7. Tener que mantener un trato directo con los familiares & 0.85 & 0.9 \\
\hline 6. Tener que mantener un trato directo con el paciente & 0.58 & 0.88 \\
\hline
\end{tabular}


descripción de las características de la muestra. Asimismo se han realizado análisis diferenciales de los factores estresantes y del burnout en función de las variables demográficas y laborales mediante la prueba $t$ de Student para muestras independientes y el análisis ANOVA de un factor. También se ha realizado un análisis de correlaciones de Pearson para conocer la relación de las variables dependientes con la edad y la experiencia laboral. Asimismo, se realizaron análisis de regresión través del método de pasos sucesivos (stepwise) con el fin de examinar qué variables ejercen una mayor influencia en los síntomas observados en la muestra.

\section{Resultados}

En el Cuestionario de Factores Estresantes, la media de puntuaciones en el ítem del nivel general de estrés fue de $1.89(D T=1.06)$. En cuanto al resto de puntuaciones (Tabla 2) el ítem más puntuado fue el 4 "sobrecarga o saturación en el trabajo", seguido del ítem 18 en el que se valoraba la falta de recursos humanos para cubrir las necesidades de la Unidad. En contraposición, los ítems que han sido valorados como menos estresantes son el hecho de tener contacto directo con los pacientes y los familiares, obteniendo el contacto con los familiares una puntuación ligeramente mayor.

Por otra parte, se analizaron los resultados de las tres subescalas del MBI con el fin de conocer el nivel de burnout de estos profesionales. La media de la subescala Agotamiento emocional fue de $21.6(D T=10.6)$, lo que indicaría según el baremo un nivel medio. De la misma manera, los profesionales presentaban un nivel medio de Despersonalización, siendo la media de 8.4 $(D T=5.5)$. Por último, en lo que respecta a la subescala de Realización personal, la media fue de $36(D T=$ 7.5), lo que indica un grado medio de Realización personal. Tras la valoración de las puntuaciones globales, se analizó la distribución de los participantes en función del baremo del cuestionario, tal y como se muestra en la Tabla 3.

Tabla 3. Frecuencias y porcentajes de los resultados del MBI en función del baremo

\begin{tabular}{cccccccc}
\hline & \multicolumn{2}{c}{ Agotamiento emocional } & \multicolumn{2}{c}{ Despersonalización } & \multicolumn{2}{c}{ Realización personal } \\
\cline { 2 - 7 } & Frecuencia & Porcentaje & Frecuencia & Porcentaje & Frecuencia & Porcentaje \\
\hline Bajo & 48 & 45,3 & 37 & 34.6 & 29 & 29,9 & 32 \\
Medio & 25 & 23.6 & 21 & 49.6 & 45.8 & 36 & 37.0 \\
Alto & 33 & 31.1 & 49 & &
\end{tabular}

Por tanto, teniendo en cuenta que el burnout supone la confluencia de niveles altos de agotamiento emocional y despersonalización así como niveles bajos de realización personal, y siguiendo el baremo del cuestionario, un 3\% de la población estudiada presentaría burnout, aunque entre el $31 \%$ y el $45 \%$ presentaría niveles elevados en alguno de sus componentes.

\section{Análisis diferenciales en función de variables sociodemográficas y laborales}

En función del género, las mujeres puntuaron más alto en la mayoría de los ítems de la escala de factores estresantes así como en su puntuación total $(M=70.24 ; D T=23.04$ en mujeres y $M=66.61 ; D T=30.98$ en hombres), sin que las diferencias fueran estadísticamente significativas en ninguno de los casos. Por lo que respecta a las distintas subescalas del cuestionario de burnout, no existieron diferencias significativas entre hombres en Agotamiento emocional y en Realización personal. Por otra parte, los hombres $(M=10.61$; $D T=5.63)$ puntuaron más en Despersonalización que las mujeres $(M=7.59 ; D T=5.29)(t(104)=2.55(p=.012)$.
Por lo que respecta al puesto laboral, en el cuestionario de factores estresantes fueron las auxiliares las que puntuaron más alto, seguidas de enfermeras y médicos, si bien las diferencias no fueron estadísticamente significativas $(t(2)=1.48 ; p=0.235)$. Tampoco se encontraron diferencias significativas en ninguna de las tres subescalas del BDI, aunque las medias muestran en los tres casos puntuaciones mayores en el caso de las auxiliares.

\section{Análisis correlacionales}

Ya que las variables de edad y experiencia profesional, tanto como profesional sanitario como trabajando específicamente en la UCI, correlacionan ampliamente entre ellas, se controlaron esas variables a la hora de realizar los análisis de correlación de Pearson. Sin embargo, estos análisis no mostraron relaciones significativas de estas dos variables con la puntuación total de factores estresantes ni con las distintas subescalas del burnout, siendo los coeficientes obtenidos próximos a cero en casi todos los casos. 


\section{Variables predictoras del estrés percibido y del burnout}

Tras realizar un análisis de regresión múltiple (Tabla 4) se observa que la variable Agotamiento emocional era predicha por la experiencia profesionaly el género, con una varianza explicada de 7.1\%. Así, según los resultados, tener mayor experiencia y ser mujer se asocian a una mayor probabilidad de sentirse agotado emocionalmente. Por lo que respecta a la Despersonalización, ésta fue predicha sólo por el género, con una varianza explicada de $5.3 \%$. En este caso, las mujeres tienen menos probabilidad de presentar síntomas de Despersonalización que los hombres. Ni la puntuación total de estrés, ni la Realización personal resultaron ser predictores significativos.

Tabla 4. Análisis de regresión: predicción de burnout

\begin{tabular}{|c|c|c|c|c|c|c|}
\hline \multirow[b]{2}{*}{ Variable dependiente } & \multirow[b]{2}{*}{ Variables predictoras ${ }^{1}$} & \multirow[b]{2}{*}{ Beta } & \multirow[b]{2}{*}{ Error } & \multicolumn{2}{|c|}{ IC $95 \%$} & \multirow[b]{2}{*}{$\%$ varianza } \\
\hline & & & & Mín. & Máx. & \\
\hline \multirow[t]{2}{*}{ Agotamiento emocional } & Experiencia en la profesión & $.233^{*}$ & .011 & .003 & .045 & 7.1 \\
\hline & Género & $-.208^{*}$ & 2.352 & -9.45 & -.096 & \\
\hline Despersonalización & Género & $.251^{*}$ & 1.24 & .619 & 5.578 & 5.3 \\
\hline
\end{tabular}

${ }^{1}$ Codificación de las variables independientes: $\operatorname{mujer}=1$, hombre $=2 ; * p<.05$.

\section{Discusión}

Los resultados de este trabajo muestran que los factores que producen más estrés a los profesionales de UCI son los relacionados con la sobrecarga de trabajo y la falta de recursos humanos para cubrir las necesidades. Además, se valoran de manera importante otros factores relacionados con el trabajo en una UCI, tal $t$ como la necesidad de tomar decisiones de manera rápida, las consecuencias que se pueden derivar de estas decisiones y la presión temporal.

Nuestros resultados coinciden con los encontrados en la literatura científica. Así, diversos estudios realizados con personal de UCI coinciden en los principales factores estresantes: la sobrecarga y la falta de personal. Igualmente, estos estudios encuentran otros factores como la sobre-exigencia, el sentirse el responsable último de la evolución del paciente, la falta de tiempo libre, los conflictos jerárquicos, la mala organización y los horarios (Coomber, et al., 2002; Macas, Kolciniene, Vetaite, Bilskiene y Mikalauskas, 2010). En cuanto al burnout, analizando los resultados de manera global vemos que los participantes de este trabajo muestran un nivel medio de Agotamiento, Despersonalización y Realización personal.

Al comparar nuestros resultados con los de trabajos previos realizados en otros países, se aprecia que la prevalencia de burnout (alto nivel de Agotamiento emocional y de Despersonalización y baja Realización personal) ha resultado ligeramente menor que la obtenida en un estudio realizado con médicos intensivistas en distintas UCIs de Francia (Embriaco et al., 2008). Si tenemos en cuenta solamente la submuestra de médicos de nuestro trabajo, vemos que las diferencias entre las puntuaciones de las dos muestras todavía son mayores.
Analizando los resultados en función de las distintas variables independientes, vemos que las mujeres presentan mayor puntuación en "Agotamiento emocional" aunque esta diferencia no resulta significativa estadísticamente, mientras que los hombres presentan significativamente mayor puntuación en "Despersonalización", y no se encuentran diferencias en "Realización personal". Los resultados van en la línea de otros estudios no específicos de UCI. Así, Grau Suñer y García (2005) concluían que el Agotamiento era mayor en mujeres, y que la Despersonalización era menor que en los hombres. Sin embargo, estos autores encontraron que la Realización personal era más baja en las mujeres que en los hombres.

Por lo que respecta a la relación entre la edad y el burnout, nuestros resultados no muestran relación entre ellas, en línea con los resultados de otros autores (Risquez, Hernández, y Fernández, 2008).

En cuanto al puesto laboral, los análisis no muestran diferencias significativas entre los grupos, estando en la línea de otros resultados encontrados en la literatura (Ordenes, 2004). Sin embargo, otros autores sí encuentran diferencias entre distintos grupos de profesionales; así por ejemplo Giannini, et al. (2013) y Atance (1997) hablan respectivamente de un mayor burnout o cansancio emocional en los enfermeros frente a los médicos especialistas. Por el contrario, Pera y Serra-Prat (2002) encuentran mayores niveles de burnout en médicos que en enfermeros.

En lo que se refiere a la antigüedad en la profesión, no se encuentra relación con ninguna de las subescalas del MBI. Tal y como ocurre en diversas variables relacionadas con el burnout, la literatura no obtiene una evidencia unánime en este aspecto. Algunos estudios (no específicos) coinciden con nuestros resultados concluyendo que la antigüedad no 
se relaciona con la vulnerabilidad al síndrome (Moreno et al., 2014). Sin embargo otros refuerzan la idea de la relación concluyendo que las personas más propensas a padecer el síndrome son aquellos que llevan menos tiempo en la profesión (Mollart, Skinner, Newing, y Foureur, 2013).

En cuanto a las variables predictoras del burnout, hemos encontrado que el género junto con la experiencia en la profesión, predicen la aparición de síntomas de Agotamiento emocional, siendo las mujeres con mayor experiencia en la profesión las que presentarían niveles más altos. En el caso de la Despersonalización, es únicamente el género el factor predictor, siendo los hombres los que presentan mayor probabilidad de experimentar síntomas relacionados con este aspecto. El papel del género en el burnout es controvertido. Algunos autores afirman que son las mujeres las que presentan mayores niveles de Agotamiento emocional (Atance, 1997), otros contrariamente, afirman que son los hombres los que presentan mayores niveles de este síndrome (Pera y Serra-Prat, 2002). Finalmente otros no detectan diferencias en función del género (Hyman, et al., 2011).

Nuestro estudio tiene algunas limitaciones. El tamaño muestral resulta insuficiente para realizar algunos análisis que nos hubiesen aportado mucha más información. Otra limitación se refiere a la relativa representatividad de la muestra ya que la participación se ha limitado a un hospital por lo que no se puede afirmar que los resultados sean indicativos de la realidad a nivel nacional. Subsanando ambas, nos permitirían concretar más sobre las relaciones entre las distintas variables estudiadas.

A pesar de estas limitaciones, pensamos que el presente estudio arroja información valiosa acerca de la necesidad de realizar programas específicos para los profesionales sanitarios de UCI que les proporcionen estrategias que permitan la prevención de aspectos que, como el burnout, pueden influir tanto en la calidad de vida de nuestros profesionales como en la calidad de los cuidados que ofrecen.

\section{Referencias}

Atance, J. C. (1997). Aspectos epidemiológicos del síndrome de burnout en personal sanitario. Revista Española de Salud Pública, 71 (3), 293-303. doi: 10.1590/s1135-57271997000300008.

Ballester, R., Gómez, S., Gil, B., y Abizanda, R. (2015) Ansiedad, depresión y malestar emocional en los profesionales sanitarios de las Unidades de Cuidados Intensivos. Anales de Psicología, 31 (2), 743-750. doi: 10.6018/analesps.31.2.158501

Bargellini, A., Barbieri, A., Rovesti, S., Vivoli, R., Roncaglia, R., \& Borella, P. (2000). Relation between immune variables and burnout in a sample of physicians. Occupational and Environmental Medicine, 57, 453-457. doi: 10.1136/oem.57.7.453.

Bresó, E., Caballero, C., y González, O. (2015). Burnout en estudiantes universitarios. Psicología Desde el Caribe, 32 (3).
Coomber, S., Todd, C., Park, G., Baxter, P., Firth-Cozens, J., \& Shore, S. (2002). Stress in UK intensive care unit doctors. British Journal of Anaesthesia, 89 (6), 873-881. doi: 10.1093/bja/ aef 273

Embriaco, N., Azoulay, E., Barrau, K., Kentish, N., Pochard, F., Loundou, A., \& Papazian, L. (2007). High level of burnout in intensivists: Prevalence and associated factors. American Journal of Respiratory and Critical Care Medicine, 175 (7), 686692. doi: 10.1164/rccm.200608-1184OC

Embriaco, N., Hraiech, S., Azoulay, E., Baumstarck-Barrau, K., Forel, J. M., Kentish-Barnes, N., Pouchard, F., Loundou, A., Roch, A., \& Papazian, L. (2012). Symptoms of depression in IC U physicians. Annals of Intensive Care, 2 (1), 1-8. doi: 10.1186/ 2110-5820-2-34

Esteras, J., Chorot, P., y Sandín, B. (2014). Predicción del Burnout en los docentes: Papel de los factores organizacionales, personales y sociodemográficos. Revista de Psicopatología y Psicología Clínica, 19 (2), 79-92.

Fernández, G. (2000). Burnout y trastornos psicosomáticos. Revista de Psicología del Trabajo y de las Organizaciones, 16, 229-235. doi: 10.1016/S2007-4719 (14)70393-X

Giannini, A., Miccinesi, G., Prandi, E., Buzzoni, C., Borreani, C., \& OD IN Study Group (2013). Partial liberalization of visiting policies and IC U staff: A before-and-after study. Intensive Care Medicine, 39 (12), 2180-2187. doi: 10.1007/S00134-013-3087-5

Gil-Monte, P. R. (1994). El síndrome de burnout: Un modelo multicausal de antecedentes y consecuentes en profesionales de enfermería. Tesis doctoral, Facultad de Psicología, Universidad de La Laguna

Gil-Monte, P. R., \& Peiró, J. M. (1999). Validez factorial del Maslach Burnout Inventory en una muestra multiocupacional. Psicothema, 11 (3), 679-689.

Gómez-Alcaina, B., Montero-Marín, J., Demarzo, M. M., Pereira, J. P., y García-Campayo, J. (2013). Utilidad de los marcadores biológicos en la detección precoz y prevención del síndrome de burnout. Revista de Psicopatología y Psicología Clínica, 18 (3), 245-253.

Grau, A., Suñer, R., y García, M. M. (2005). Desgaste profesional en el personal sanitario y su relación con los factores personales y ambientales. Gaceta sanitaria, 19 (6), 463-470. doi: 10.1016/ S0213-9111 (05)71397-2

Grossi, G., Perski, A., Evengard, B., Blomkvist, V., \& Orth-Gomér, K. (2003). Physiological correlates of burnout among women. Journal of Psychosomatic Research, 55, 309-316. doi: 10.1016/ S0022-3999 (02)00633-5

Hyman, S. A., Michaels, D. R., Berry, J. M., Schildcrout, J. S., Mercaldo, N. D., \& Weinger, M. B. (2011). Risk of burnout in perioperative clinicians. Anesthesiology, 114 (1), 194-204. doi: 10.1097/AL N. 0b013e318201ce9a

Jiménez, B. M., Lara, R. M. M., Muñoz, A. R., Chavez, A. P., y Loo, M. M. M. (2014). El síndrome de burnout en una muestra de psicólogos mexicanos: Prevalencia y factores sociodemográficos asociados. Psicología y Salud, 16 (1), 5-13.

Macas, A., Kolciniene, A., Vetaite, I., Bilskiene, D., \& Mikalauskas, A. (2010). Stress and fatigue among anaesthesia and intensive care doctors in Lithuania. Critical Care, 14 (Suppl 1), 1-1. doi: $10.1186 / \mathrm{cc} 8233$

Maslach, C., \& Jackson, S. (1986). Maslach Burnout Inventory Manual. Palo Alto California: Consulting Psychology Press. 
Mollart, L., Skinner, V. M., Newing, C., \& Foureur, M. (2013). Factors that may influence midwives work-related stress and burnout. Women and Birth, 26 (1), 26-32. doi: 10.1016/j.wombi.2011.08.002.

Moreno, M. P., Bermúdez, D., Beltrán, C. A., Castellanos, J. P., Salinas, E. F., y Pérez, G. A. (2014). Prevalencia de estrés y burnout en los trabajadores de la salud en un hospital ambulatorio. Psicología y Salud, 13 (1), 47-52.

Ordenes, N. (2004). Prevalencia de Burnout en trabajadores del hospital Roberto del Río. Revista Chilena de Pediatría, 75 (5), 449-454. doi: 10.4067/S0370-41062004000500006.

Ortega, C., y López, F. (2004). El burnout o síndrome de estar quemado en los profesionales sanitarios: revisión y perspectivas. International Journal of Clinic and Health Psychology, 4, 137160. IS SN 1576-7329.

Pera, G., y Serra-Prat, M. (2002). Prevalencia del síndrome del quemado y estudio de los factores asociados en los trabajadores de un hospital comarcal. Gaceta Sanitaria, 16 (6), 480-486. doi: 10.1016/S0213-9111 (02)71968-7

Poncet, M. C., Toullic, P., Papazian, L., Kentish-Barnes, N., Timsit, J. F., Pochard, F., Chevret, S., Schlemmer, B., \& Azoulay, E. (2007). Burnout syndrome in critical care nursing staff. American Journal of Respiratory and Critical Care Medicine, 175 (7), 698-704. doi: 10.1164/rccm.200606-806OC

Quenot, J. P., Rigaud, J. P., Prin, S., Barbar, S., Pavon, A., Hamet, M., Jacquiot, N., Hervé, C., Charles, P. E. \& Moutel, G. (2012).
Suffering among carers working in critical care can be reduced by an intensive communication strategy on end-of-life practices. Intensive Care Medicine, 38 (1), 55-61. doi: 10.1007/s00134011-2413-z

Raggio, B., \& Malacarne, P. (2007). Burnout in intensive care unit. Minerva Anestesiology, 73, 195-200.

Reader, T. W., Cutherson, B. H., \& Decruyanier, J. (2008). Burn-out in the IC U, potential consequences and patient well-being. Intensive Care Medicine, 34, 4-6. doi: 10.1007/s00134-007-0907-5

Richart, M., Cabrero, J., y Reig A. (1993). Hospitalización y estrés en el paciente, percepción diferencial de estresores entre paciente y personal de enfermería. Análisis y Modificación de Conducta, 19, 75-89. IS SN 0211-7339

Risquez, M. R., Hernández, F. P., y Fernández, C. G. (2008). Burnout y salud percibida en profesionales de enfermería de Cuidados Intensivos. Enfermería Intensiva, 19 (4), 169-178.

Santos, M. D. L. M., y Abalo, J. G. (2014). Síndrome de Burnout en médicos que trabajan en unidades de cuidados intensivos neonatales. Psicología y Salud, 15 (1), 25-32.

Sexton, J. B., Thomas, E. J., \& Helmreich, R. L. (2000). Error, stress, and teamwork in medicine and aviation: cross sectional surveys. BM J, 320 (7237), 745-749. doi: 10.1136/bmj.320. 7237.745

Verdon, M., Merlani, P., Perneger, T., \& Ricou, B. (2008). Burnout in a surgical IC U team. Intensive Care Medicine, 34, 152-156. doi: 10.1007/S00134-007-0907-5 